\title{
Research and Implementation of high accuracy timing method based on GPS PPS in spaceborne remote sensing cameras
}

\author{
Pan Wei-jun *, Liu Tao, He Qiang-min, Xiao Long, Wang Yan, Zhang Ye \\ Beijing Institute of Space Mechanics \& Electricity, Beijing 100094, China \\ jandyycool@163.com
}

Key words: Remote sensing, Imaging moment, Timing accuracy, GPS PPS

Abstract. As requirements of stereo-positioning accuracy of high resolution remote sensing cameras becoming higher and higher, the timing accuracy of imaging should be within a few micro-seconds. However, imaging timing accuracy is easily influenced by frequency drift due to aging of clock sources and temperature variation. In this paper, a high accuracy timing method based on GPS PPS is proposed. According to the timing accuracy analysis, the proposed timing method has higher accuracy compared with conventional timing method. The timing method is illustrated in detail and test results show that the optimal timing method can improve timing accuracy up to higher level which can be used in the field of high resolution remote sensing cameras.

\section{Introduction}

With the development of space optical and electrical technologies, high- resolution is becoming the main feature of remote sensing cameras. For example, the resolutions of commercial imaging satellites -Worldview-2 and GeoEye- 1 are up to $0.46 \mathrm{~m}$ and $0.41 \mathrm{~m}$ respectively. Furthermore, high accuracy geometric geo-positioning is the basis and vital technology for different applications using HRSI (High-Resolution Satellite Imagery). So improving the geo-positioning accuracy is the significant task of remote sensing.

According to the rigorous sensor model of linear push-broom cameras as depicted in fig. 1, in order to get the rigorous geometric imaging function ${ }^{[1]}$, the platform parameters 、 camera parameters vorbital parameters and attitude parameters on the imaging moment must be measured and calculated. As shown in fig.1, the orbital data imaging data attitude data and measured platform vibration data should be unified with GPS observation time.

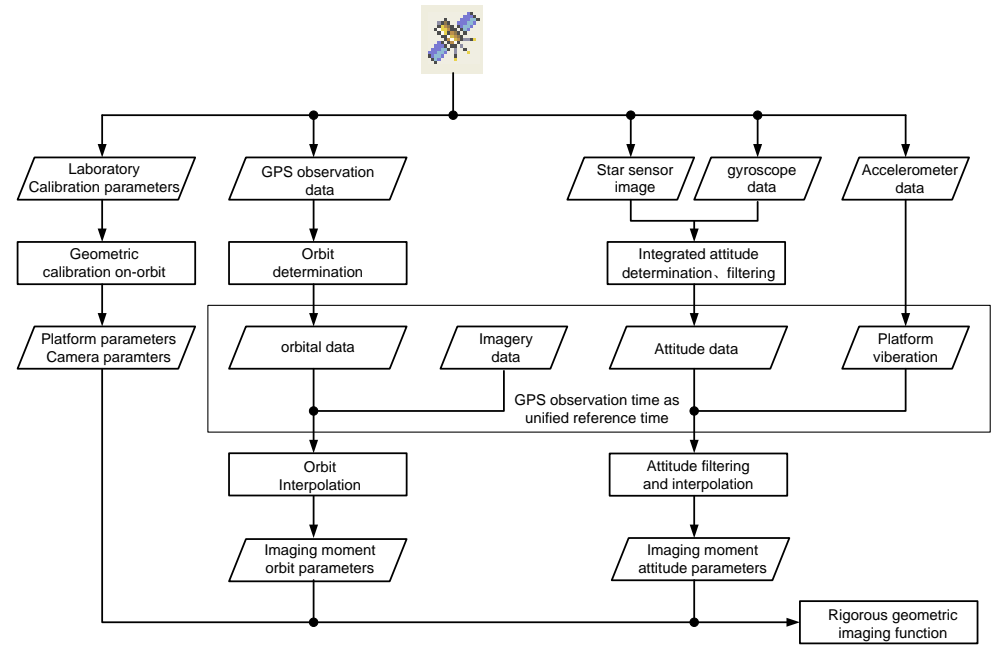

Fig.1 rigorous sensor model of linear push-broom camera

However, the GPS reference time is refreshed in a long time period compared with the period of imaging line synchronal signal. The GPS observation time is refreshed every second while the 
imaging line synchronal signal period is down to less than 100 micro-seconds in conventional high resolution applications. The refreshing timing graph of GPS observation time and imaging line synchronal signal is shown as fig.2.

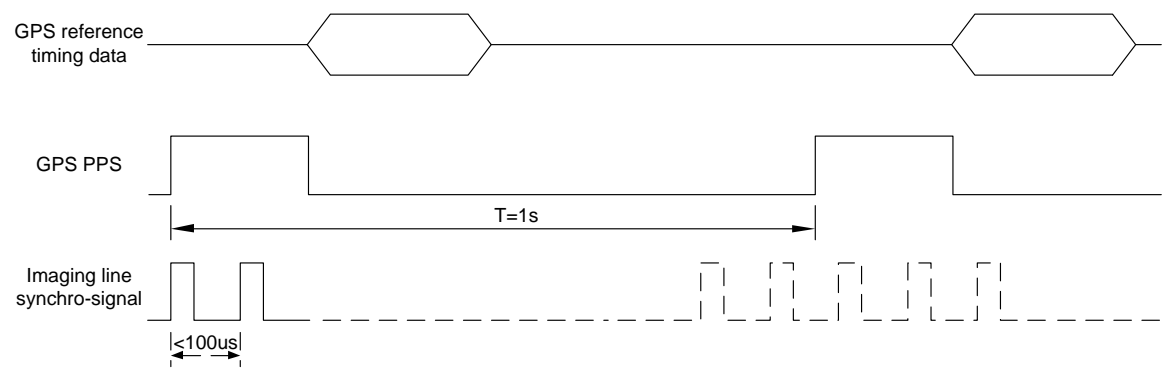

Fig.2 refreshing timing of GPS observation time and imaging line synchronal signal

In order to get the time of every rising edge of line synchronal signal, interpolation method should be adopted. A counter driven with local oscillator is used as a timing component. The counter data will be registered at the every rising edge of the line synchronal signal and then the imaging time will be inferred with the local oscillator frequency. However, the local oscillator frequency is influenced easily by the aging of clock sources and temperature variation. So the accuracy of imaging timing is affected by the stability of local oscillator frequency ${ }^{[2]}$.

In this paper, a high accuracy timing method is proposed which eliminates the factor of frequency drift caused by the aging and the temperature shift. Firstly, the timing accuracy will be analyzed and compared between the conventional method and the proposed method. And then, the implementation of the proposed method will be described in detail. Finally, the test results show that an excellent timing accuracy is obtained with the proposed method.

\section{Timing accuracy analysis}

There are two interpolation methods in the application of imaging timing. One is the conventional timing method which ignores the frequency drift and takes the frequency as fixed and accurate parameter. The other method is called frequency drift compensation method which measures the actual frequency of the local oscillator ${ }^{[3]}$. The difference between the two methods is that the local counter resets at the every rising edge of the GPS PPS with the conventional method while the local counter will be registered at rising edge of both the line synchronal signal and the rising edge of GPS PPS with the frequency drift compensation method. Timing accuracy of the two different method will be analyzed and compared as below.

Timing accuracy analysis of conventional timing method. The timing graph of conventional timing method is depicted in Fig.3. Local counter resets at the rising edge of GPS PPS.

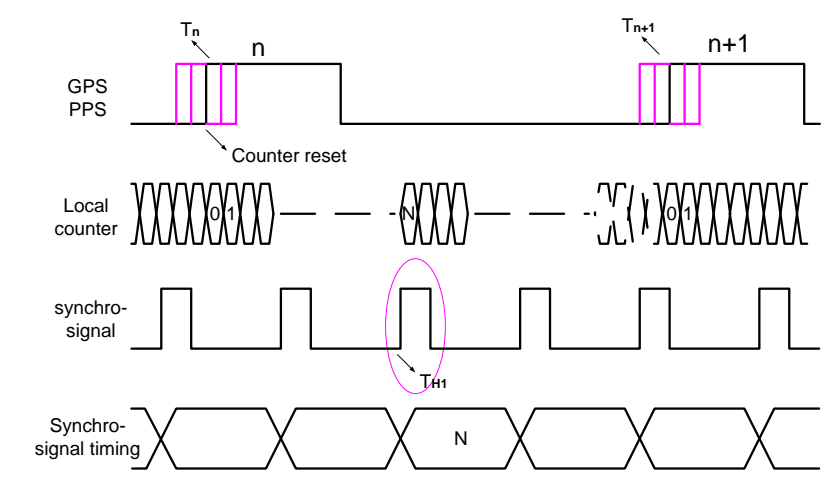

Fig.3 Timing graph of conventional timing method 
Presume GPS PPS $n$ indicates the GPS reference time is $T_{n}$. In order to infer the imaging time data $\mathrm{T}_{\mathrm{H} 1}$ of a certain rising edge of line synchronal signal, the counter data $\mathrm{N}$ registered at the observed rising edge and the local oscillator frequency $f$ will be used ${ }^{[4]}$.

The imaging time value $\mathrm{T}_{\mathrm{H} 1}$ is formalized as :

$$
T_{H 1}=T_{n}+\frac{N}{f}
$$

The timing deviation $\Delta \mathrm{T}_{\mathrm{H} 1}$ is inferred as:

$$
\Delta T_{H 1} \leq\left|\frac{\Delta N}{f}\right|+\left|\frac{N}{f} \frac{\Delta f}{f}\right|
$$

Timing accuracy analysis of frequency drift compensation timing method.The timing graph of frequency drift compensation timing method is depicted in Fig.4. The local counter continues counting up during the whole imaging period ${ }^{[5]}$. At the rising edges of both GPS PPS and line synchronal signal, the local counter data are registered. Presume the GPS reference time data and registered local counter data of GPS PPS $k$ are $T_{K}$ and $N_{K}$ respectively. GPS PPS $n$ and GPS PPS $(n+m)$ are taken into consideration. According to the presumption above, the GPS reference time data and registered local counter data of GPS PPS $n$ are $T_{n}$ and $N_{n}$ respectively. In the same way, the GPS reference time data and registered local counter data of GPS PPS $(n+m)$ are $T_{n+m}$ and $\mathrm{N}_{n+m}$ respectively. With the registered local counter data $N_{n}$ of GPS PPS $n$ and the registered local counter data $\mathrm{N}_{n+m}$ of GPS PPS $(n+m)$, actual oscillator frequency $\mathrm{f}^{\prime}$ can be inferred as $\mathrm{f}^{\prime}=\frac{\mathrm{N}_{\mathrm{n}+\mathrm{m}}-\mathrm{N}_{\mathrm{n}}}{\mathrm{m}}$.

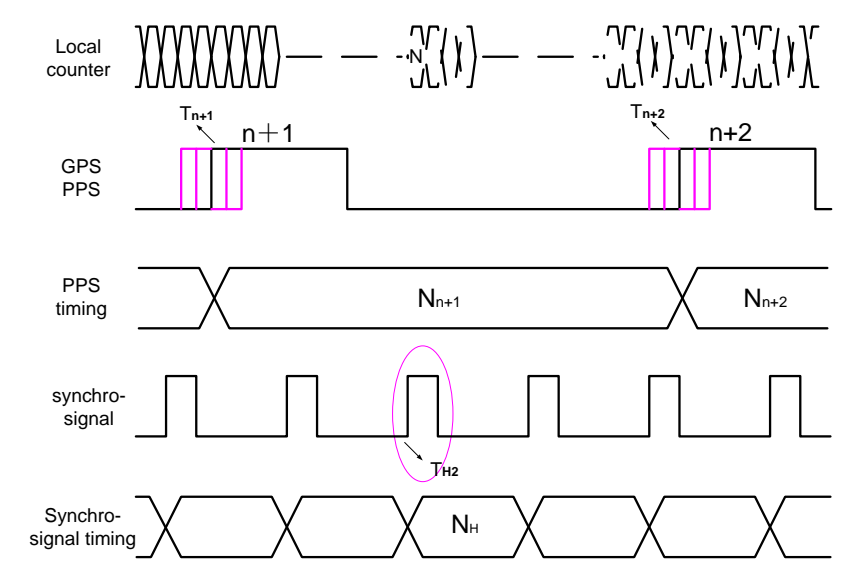

Fig.4 Timing graph of frequency drift compensation timing method

The timing value $\mathrm{T}_{\mathrm{H} 2}$ of a certain rising edge of line synchronal signal at some position between the GPS PPS $(n+m)$ and the GPS PPS $(n+m+1)$ can be calculated as follows

$$
T_{H 2}=T_{n+m}+\frac{N_{H}-N_{n+m}}{f^{\prime}}
$$

Where $\mathrm{N}_{\mathrm{T}}=\mathrm{N}_{\mathrm{H}}-\mathrm{N}_{\mathrm{n}+\mathrm{m}}$, so formulation (3) can be transformed as follows

$$
T_{H 2}=T_{n+m}+\frac{N_{T}}{f^{\prime}}
$$

The timing deviation $\Delta \mathrm{T}_{\mathrm{H} 2}$ is inferred as:

$$
\Delta T_{H 2} \leq\left|\frac{\Delta N_{T}}{f^{\prime}}\right|+\left|\frac{N_{T}}{f^{\prime}} \frac{\Delta f^{\prime}}{f^{\prime}}\right|
$$

Analysis of deviation parameters. According to the timing deviation formulation (2) of the conventional timing method and the timing deviation formulation (5) of frequency drift compensation timing method, the timing deviation is mainly influenced with two factors. One is counting deviation like $\Delta \mathrm{N}$ or $\Delta \mathrm{N}_{\mathrm{T}}$, the other is oscillator frequency drift like $\Delta \mathrm{f}$ or $\Delta \mathrm{f}^{\prime}$.

The counting deviation is affected by the propagation delay drift of the GPS PPS. The GPS PPS propagation chain is depicted as Fig.5(a).The GPS PPS propagation chain includes 3 different kinds of delay ${ }^{[6]}$. One is on-boarding trace propagation delay which is only hundreds of picoseconds. The other is cable propagation delay between boards which is tens of nanoseconds. The third is chip 
propagation delay which is a few nanoseconds. So he combinational delay is less than 1 microsecond. If local oscillator frequency is $1 \mathrm{MHz}$, the counting deviation can be inferred as follows

$$
\Delta N=\Delta N_{T}=1
$$

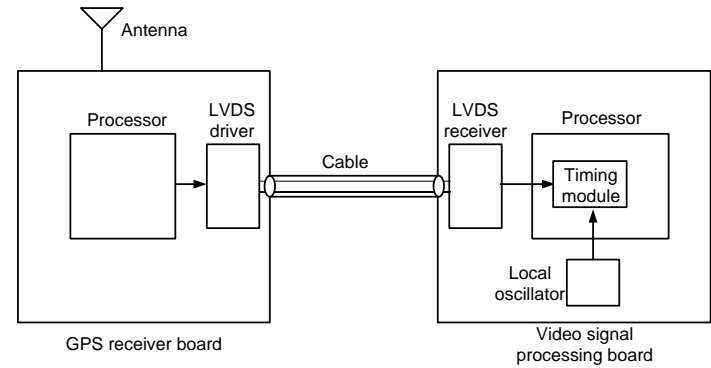

(a) Scheme of GPS PPS Propagation chain

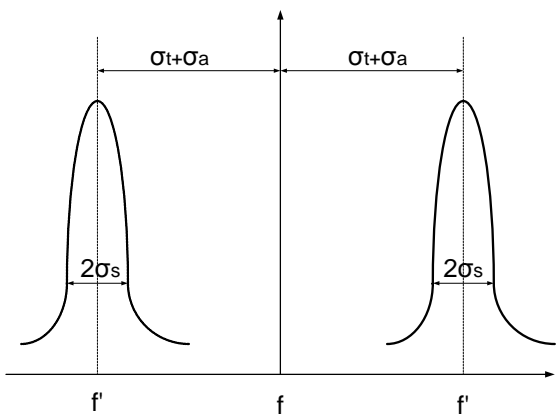

(b)Frequency drift model of local oscillator

Fig.5 Model scheme of deviation parameter

The oscillator frequency drift model is shown in Fig.5(b). The frequency drift is composed with 3 kinds of deviation parameters. The parameter $\sigma_{t}$ indicates frequency drift caused by the temperature variation. The parameter $\sigma_{\mathrm{a}}$ indicates frequency drift caused by the aging of local oscillator and the parameter $\sigma_{\mathrm{s}}$ is for the random jitter.

As for the frequency drift compensation timing method, the frequency drift will be remarkably smaller than the conventional timing method as a result of real-time measuring oscillator frequency. The frequency drift caused by the temperature variation and the aging of the local oscillator will be eliminated with the proposed timing method. So, the oscillator frequency drift of the conventional timing method and the frequency drift compensation timing method are formulized as follows respectively.

$$
\begin{aligned}
& \Delta \mathrm{f}=\sigma_{\mathrm{a}}+\sigma_{\mathrm{t}}+\sigma_{\mathrm{s}} \\
& \Delta \mathrm{f}^{\prime}=\sigma_{\mathrm{s}}
\end{aligned}
$$

For example, for one local oscillator, $\mathrm{f}=1 \mathrm{MHz}, \sigma_{\mathrm{t}}=40 \mathrm{ppm}, \sigma_{\mathrm{a}}=20 \mathrm{ppm}$ and $\sigma_{\mathrm{s}}=$ $10 \mathrm{ppm}$.According to formulation $(2)(5)(6)(7)(8)$, the timing deviation for the conventional timing method and the frequency drift compensation timing method can be calculated as below:

$$
\begin{gathered}
\Delta \mathrm{T}_{\mathrm{H} 1} \leq 1+40+20+10=71 \mu \mathrm{s} \\
\Delta \mathrm{T}_{\mathrm{H} 2} \leq 1+10=11 \mu \mathrm{s}
\end{gathered}
$$

As we can see from above, the proposed method has a remarkably higher timing accuracy than the conventional timing method.

\section{Implementation of high accuracy timing method}

Designing of the high accuracy timing circuit. In this section, the frequency drift compensation timing circuit will be described in detail. The functional scheme of high accuracy timing circuit is depicted as shown in figure 6.

The timing circuit is composed with 4 different function modules-local counter, edge detection module, timing data registers and imaging timing algorithm module. The function of such modules as mentioned above will be described as follows ${ }^{[7]}$ :

(a)Local counter, which is driven by local oscillator, keeps counting up during the whole imaging period. The counter data is the time interpolation between the two neighboring GPS PPS;

(b)Edge detection module, which is used for detecting the ring edge of both GPS PPS and line synchronal signal and generating the ring edge signals for both of them; 


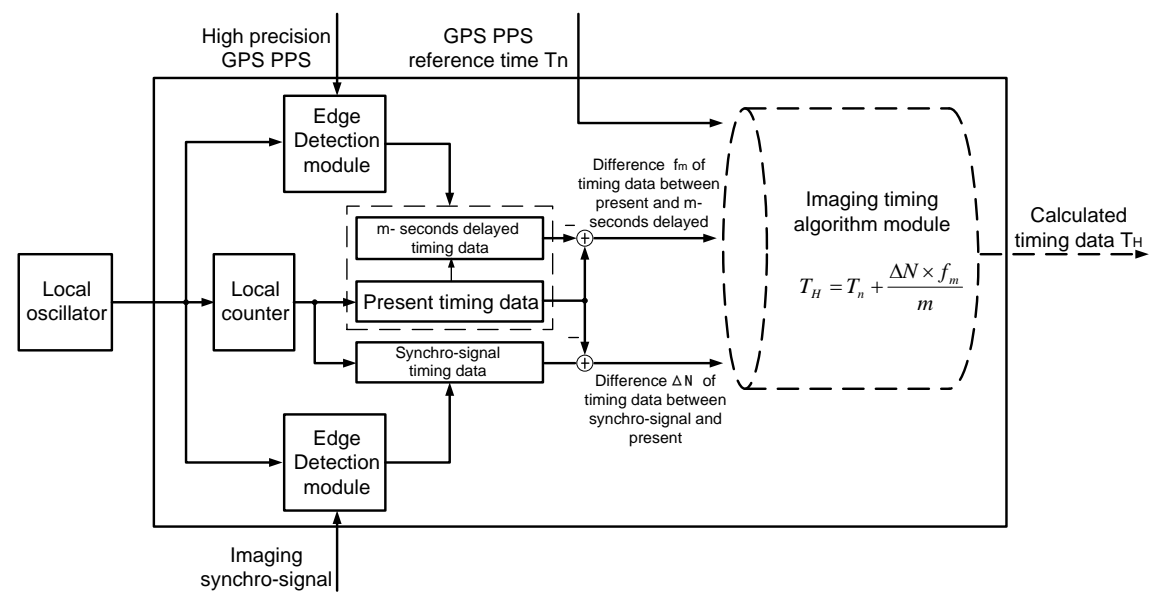

Fig.6 Functional scheme of frequency drift compensation timing circuit

(c)Timing data registers. There are 3 registers in the designed timing circuit. One is used for registering the timing data of line synchronal signal rising edge. The other two registers are used for registering the timing data of GPS PPS rising edge and $\mathrm{m}$ seconds delayed GPS PPS rising edge;

(d)Imaging timing algorithm module is used for calculating the timing data. With the difference value between the resent timing data and $\mathrm{m}$-seconds delayed timing data and the difference value $\Delta \mathrm{N}$ between the line synchronal signal timing data and the present GPS PPS timing data, the imaging timing data can be calculated as: $T_{H}=T_{n}+\frac{\Delta N \times f_{m}}{m}$.

Simulation of the high accuracy timing circuit. The proposed timing circuit is designed with VHDL and implemented with FPGA platform. To verify the functional validation, the simulation tool ModelSim is used for functional simulation. The simulation result of the proposed timing circuit is shown in Figure 7.

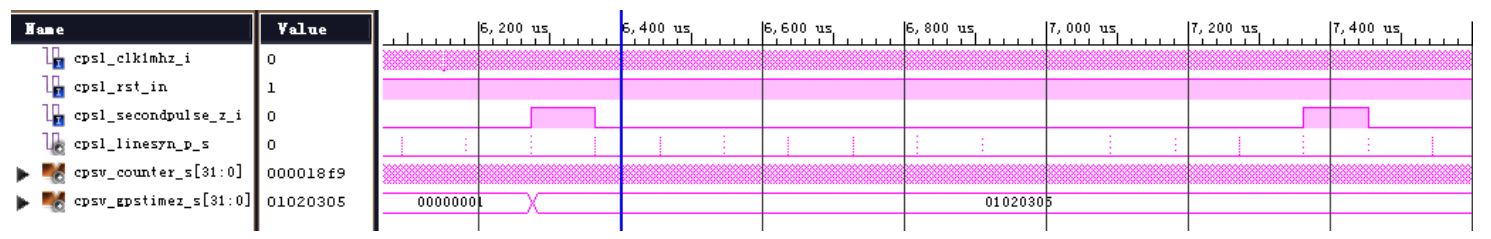

Fig.7 Simulation result of frequency drift compensation timing circuit

As seen from figure 7, the high accuracy timing circuit performs valid functional operation.

\section{Tests and results}

To verify the performance of the frequency drift compensation timing method, the circuit is put in the temperature-controllable box. Through adjusting the ambient temperature, the timing deviations of different timing modes are measured. The data acquired in different temperature from $-30^{\circ} \mathrm{C}$ to $60^{\circ} \mathrm{C}$ and different timing modes are listed in table 1 .

Tab.1 Timing deviation measured data list

\begin{tabular}{|c|c|c|}
\hline & Conventional timing method & Proposed timing method \\
\hline$-30^{\circ} \mathrm{C}$ & $32 \mu \mathrm{s}$ & $6 \mu \mathrm{s}$ \\
\hline$-20^{\circ} \mathrm{C}$ & $26 \mu \mathrm{s}$ & $5 \mu \mathrm{s}$ \\
\hline$-10^{\circ} \mathrm{C}$ & $21 \mu \mathrm{s}$ & $5 \mu \mathrm{s}$ \\
\hline $0^{\circ} \mathrm{C}$ & $17 \mu \mathrm{s}$ & $6 \mu \mathrm{s}$ \\
\hline $10^{\circ} \mathrm{C}$ & $10 \mu \mathrm{s}$ & $7 \mu \mathrm{s}$ \\
\hline
\end{tabular}




\begin{tabular}{|l|c|c|}
\hline $20^{\circ} \mathrm{C}$ & $7 \mu \mathrm{s}$ & $5 \mu \mathrm{s}$ \\
\hline $30^{\circ} \mathrm{C}$ & $-9 \mu \mathrm{s}$ & $6 \mu \mathrm{s}$ \\
\hline $40^{\circ} \mathrm{C}$ & $-15 \mu \mathrm{s}$ & $4 \mu \mathrm{s}$ \\
\hline $50^{\circ} \mathrm{C}$ & $-21 \mu \mathrm{s}$ & $5 \mu \mathrm{s}$ \\
\hline $60^{\circ} \mathrm{C}$ & $-27 \mu \mathrm{s}$ & $6 \mu \mathrm{s}$ \\
\hline
\end{tabular}

As we can see from table 1, the frequency drift compensation timing method utterly eliminates the factor of temperature shift. As a result, the timing accuracy is much higher than the conventional timing method.

\section{Conclusion}

An oscillator frequency drift compensation timing method which can eliminate the frequency drift caused by the temperature variation and the aging of oscillator is proposed in this paper. Timing accuracy analysis is made and compared and the key point of the timing circuit designing is also present. Functional Simulation and tests result exhibit that the proposed high accuracy timing method can be applied easily and reliably in the field of spaceborne remote sensing cameras.

\section{Reference}

[1] Chen, T., Shibasaki, R. and Lin, Z.. A rigorous laboratory calibration method for interior orientation of an airborne linear push-broom camera. Photogrammetric Engineering \& Remote Sensing, 2007, 73(4):369-374.

[2] Fraser,C.S., Hanley,H.B., Yamakawa,T.. Sub-meter geopositioning with IKONOS GEO imagery. Proceedings of Joint ISPRS Workshop on High Resolution Mapping from Space, Hanover, 2001.

[3] KOPPANG P, WHEELER P. Working applition of TWSTT for high precision remote synchronization $[\mathrm{A}]$. Proceedings of the IEEE International Symposium on Frequency Control[C],1998:273-277.

[4] REN Yaan, WANG Peng, XU Weidong, et al. Development and techniques of direct GPS $\mathrm{P}(\mathrm{Y})$-code acquisition[J]. GNSS World of China, 2003.(2): 2-7. (in Chinese)

[5] Wang Feng, Fu Jianhua, Chen Ligang, Zhang Xiangrong. Enhancing the GPS Clock's Time Pulse Precision By Adopting Synchronons Timing[J]. JOURNAL OF SEISMOLOGICAL RESEARCH, 2003, 26(3): 282-285

[6] L. X. Qiao, C. Yuan, L. Shuang. Improvement of precise time synchronization algorithm based on IEEE1588 [A].

Proceedings of the International Conference on Computer, Mechatronics, Control and Electronic Engineering [C]. Changchun, China, Aug. 2010, 1:pp.70-73

[7] Hu Zhihui, Zhou Yu. Research and Implementation of Hardware Timestamp Based on IEEE1588 Protocol. International Conference on Computational and Information Sciences [C]. 1586-1589.(2013). 